Maria L. Scarbrough, MD

Preeti Dalawari, MD, MSPH

Cindy C. Bitter, MD

St. Louis, $M O$

\section{Pharmacologic Prophylaxis for Acute Mountain Sickness: A Systematic Shortcut Review}

Background.-Since the 1970s, multiple studies have explored pharmacologic interventions to prevent acute mountain sickness (AMS). A systematic review of this topic published in 2000 found that both acetazolamide and dexamethasone were effective in preventing the development of AMS. Since 2000, a number of other agents have been reported to be beneficial.

Objective.- Perform a systematic shortcut review to determine which agents safely and effectively prevent the development of AMS in adult patients who rapidly ascend to high altitudes.

Methods.-We searched MEDLINE, Embase, the Cochrane Central Register of Controlled Trials, SPORTDiscus, Emergency Medical Abstracts, and ClinicalTrials.gov from the year 2000 to July 2011. Only randomized, placebo-controlled trials (RCT) with an $\mathrm{N} \geq 50$ and systematic reviews (SR) were reviewed. Standard criteria for assessing trial quality were independently assessed by two authors.
Results.-786 citations were retrieved of which 105 were reviewed. Of these, 12 met inclusion criteria including $1 \mathrm{SR}$ and 11 RCTs; however, 4 RTCs were excluded for high risk of bias. The remaining 7 RCTs investigated antioxidants, sumatriptan, gabapentin, acetazolamide, ginkgo biloba, and magnesium. No trials studying dexamethasone met our criteria. Acetazolamide ( $250 \mathrm{mg}$ to $750 \mathrm{mg}$ daily) was associated with a reduction in AMS symptoms with a number needed to treat (NNT) ranging from 3 to 8 in 3 trials. Sumatriptan $(50 \mathrm{mg}$ daily) showed benefit in one trial with an NNT $=4$ as did gabapentin (600 mg daily) with an NNT $=6$. Antioxidants, ginkgo biloba, and magnesium were not efficacious. Significant adverse events included somnolence with gabapentin and paresthesias with acetazolamide. The SR affirmed our results but did not capture trials studying antioxidants, sumatriptan, gabapentin, or magnesium.

Conclusions.- Acetazolamide continues to be effective for the prevention of AMS, but may be associated with paresthesias. Sumatriptan and gabapentin are beneficial, but require more study.

Rawle A. Seupaul, MD Julie L. Welch, MD

Sarah T. Malka, MD Thomas W. Emmett MD, MLS Indianapolis, IN 